\title{
Solution of Weighted Residual Problems by using Galerkin's Method
}

\author{
J. Evangeline Cicelia \\ Department of Mathematics, Bharath Institute of Science \& Technology, \\ Bharath University, Selaiyur, Chennai -600073,Tamil Nadu, India evangelinecicelia@gmail.com
}

\section{Abstract}

For simple one-dimensional problems, direct stiffness method is limited. PMPE (Principle of Minimum Potential Energy) is limited to potential problems and FEM (Finite Element Method) can be applied to many engineering problems that are governed by a differential equation. Hence, there are systematic approaches, needed to generate FE equations, such as Weighted Residual method, Energy method and ordinary differential equation (second-order or fourth-order). Weighted residual method can be solved, in particular using Galerkin's method.

Keywords: Residual, Weighted, Stiffness, FEM, FE, Galerkin's Method, Differential Equations

\section{Introduction}

The theory behind finite difference solutions is intuitive and these solutions are easy to understand and straightforward to implement. However, the finite difference technique is most appropriate for problems where the computational domain is regularly shaped and the nodes are positioned in a regular pattern. The finite element technique is an alternative numerical method. The finite element technique is much less intuitive than the finite difference technique to most engineers, and the underlying theory is more involved.

However, once the theory has been developed, the finite element technique can be applied as easily to irregular geometries with an arbitrary arrangement of nodes as it can be to a regular geometry with a regular pattern of nodes. The simple approach of Galerkin's method is discussed as follows:

\subsection{Procedure}

1. Guess a solution to the differential equation, which satisfies the boundary conditions.

2. The trial solution will contain certain parameters, which can be adjusted to minimize the errors so that the trial solution is as close to the exact solution as feasible.

3. Consider the boundary value problem $y^{\prime \prime}=f\left(y, y^{\prime}, x\right)=$ $f(x, y, y)$ with boundary conditions $y(a)=A$ and $y(b)=B$

4. Write the differential equation $\mathrm{R}=\bar{y}^{\prime \prime}-f(x, \bar{y}, \bar{y})$ (ii) where, $\mathrm{R}$ is the residual of the equation when $\mathrm{R}=0$ for the exact solution $y(x)$ only which will satisfy the boundary conditions.

5. Consider the trial solution $\bar{y}(x)=C_{1} \varnothing_{1}(x)+$ when $\bar{y}(a)=A$ and $\bar{y}(b)=B$

$$
C_{2} \varnothing_{2}(x)
$$

6. Differentiate trial solution (iii) twice and substitute in (ii)

7. To find $C_{1}, C_{2}, \ldots \ldots$ weight the residual by trial functions $\emptyset_{1}(x), \emptyset_{2}(x), \ldots \ldots \ldots$ and set the integrals to zero.

$$
\therefore \int_{c} R \phi_{1}(x) d x=0, \quad \int_{c} R \phi_{2}(x) d x=0, \ldots \ldots
$$

8. Solve simultaneous equations to find the unknowns, substitute these unknowns in the appropriate solution, $\bar{y}(x)$ is obtained.

9. By using this procedure carefully the following steps, many weighted residual problems can be solved. Here, we discussed a sample solved problem, which gives the approximate solution of the problem and comparison with exact solution.

*Author for correspondence 


\section{Problem}

Use Galerkin's methods to solve the boundary value problem $y^{\prime \prime}-y+x=0,0 \leq x \leq 1, y(0)=0$ and $\mathrm{y}(1)=0$. Compare the approximate solution with exact solution.

\section{Solution}

STEP 1: The given differential equation is $y^{\prime \prime}-y+x=0$, $0 \leq x \leq 1$.

STEP 2: Consider the residual $\mathrm{R}$,

$$
R=\bar{y}^{\prime \prime}-\bar{y}+x
$$

STEP 3: To find the trial solution, which satisfies the boundary conditions, derive lagrangian polynomial which passes throw the points,

$x: 0 \frac{1}{2} 1$

$y: 0 \subset 0$

STEP 4: The resulting polynomial is

$$
\begin{aligned}
\bar{y}(x) & =4 C x(1-x) \\
& =C_{1} \cdot x(1-x) \quad \text { Where } C_{1}=4 C
\end{aligned}
$$

Since the trial solution is $\bar{y}(x)=C_{1} \phi_{1}(x)+C_{2} \phi_{2}(x) \ldots \ldots \ldots$

Where $\phi(x)=x(1-x)$

STEP 5: Differentiate twice (ii) w.r.t $x$, we get

$$
\begin{aligned}
\frac{d \bar{y}(x)}{d x} & =\frac{d}{d x}[4 C x(1-x)] \\
& =4 C[(1)(1-x)+x(-1)] \\
& =4 C[1-x-x] \\
\bar{y}^{\prime}(x) & =4 C(1-2 x)
\end{aligned}
$$

Again differentiating this, we get

$$
\begin{aligned}
& \bar{y}^{\prime}(x)=4 C(-2) \\
& \bar{y}^{\prime \prime}(x)=-8 C
\end{aligned}
$$

STEP 6: substitute the values of $\bar{y}^{\prime}(x)$ and $\bar{y}(x)$ in equation (i), we get

$$
\begin{aligned}
R & =-8 C-4 C x(1-x)+x \\
& =-8 C-4 C x+4 C x^{2}+x \\
R & =4 C x^{2}+(1-4 C) x-8 c
\end{aligned}
$$

STEP 7: WKT $\int R . \phi(x) d x=0$

i.e. $\quad \int_{0}^{1}\left(4 C x^{2}+(1-4 C) x-8 C\right)(x(1-x)) d x=0$

$\int_{0}^{1}\left(4 C x^{2}+4 C x+x-8 c\right)\left(x-x^{2}\right) d x=0$

$\int_{0}^{1}\left(4 C x^{3}-4 C x^{4}-4 C x^{2}+4 C x^{3}+x^{2}-x^{3}-8 C x+8 C x^{2}\right) d x=0$

Integrating we get,

$\frac{4 C x^{4}}{4}-\frac{4 C x^{5}}{5}-\frac{4 C x^{3}}{3}+\frac{4 C x^{4}}{4}+\frac{x^{3}}{3}-\frac{x^{4}}{4}-\frac{8 C x^{2}}{2}+\frac{8 C x^{3}}{3}=0$

$2 C x^{4}-\frac{4 C x^{5}}{5}+\frac{4 C x^{3}}{3}+\frac{x^{3}}{3}-\frac{x^{3}}{4}-4 C x^{2}=0$

Substitute the limits 0 to 1 , we get

$$
\begin{aligned}
& 2 C-\frac{4 C}{5}+\frac{4 C}{3}+\frac{1}{3}-\frac{1}{4}-4 C=0 \\
& C\left(2-\frac{4}{5}+\frac{4}{3}-4\right)+\left(\frac{1}{3}-\frac{1}{4}\right)=0 \\
& C\left(-\frac{4}{5}+\frac{4}{3}-2\right)+\left(\frac{1}{3}-\frac{1}{4}\right)=0 \\
& C\left(\frac{-12+20-30}{15}\right)+\left(\frac{4-3}{12}\right)=0 \\
& C\left(\frac{-22}{15}\right)=-\left(\frac{1}{12}\right) \\
& C=\frac{15}{12(22)} \\
& C=\frac{5}{88}
\end{aligned}
$$

STEP 8: Substitute this $C$ value in equation (ii), the approximate solution is

$$
\begin{aligned}
& \bar{y}(x)=4 \frac{5}{88} x(1-x) \\
& \bar{y}(x)=\frac{5}{22} x(1-x)
\end{aligned}
$$

STEP 9: To find exact solution

Let the given differential equation be,

$$
\begin{aligned}
& y^{\prime \prime}-y+x=0 \\
& y^{\prime \prime}-y=-x
\end{aligned}
$$

i.e. $\left(D^{2}-1\right) y=-x$

Auxiliary equation is, $m^{2}-1=0$ 


$$
m^{2}=1 \quad m= \pm 1, \quad m=1, \quad m=-1
$$

The roots are real and different

$\therefore$ The complementary function is,

$$
\text { C.F }=A e^{m_{1} x}+B e^{m_{2} x}
$$

i.e. $y=C_{1} e^{x}+C_{2} e^{-x}$

Particular integral

$$
\begin{aligned}
& P . I=\frac{1}{\left(D^{2}-1\right)(-x)} \\
& =\frac{1}{-\left(1-D^{2}\right)}(-x)=\left(1-D^{2}\right)^{-1}(x) \\
& =\left(1+D^{2}+D^{4}+\cdots\right)(x) \\
& \text { P.I }=x
\end{aligned}
$$

General solution: C.F + P.I

$$
y=C_{1} e^{x}+C_{2} e^{-x}+x
$$

Since $y(0)=0$ and $y(1)=0$

$$
\begin{aligned}
& 0=C_{1} e^{0}+C_{2} e^{0}+0 \Rightarrow C_{1}+C_{2}=0 \Rightarrow C_{2}=-C_{1} \\
& 0=C_{1} e^{1}+C_{2} e^{-1}+1 \Rightarrow C_{1} e+\left(-C_{1}\right) e^{-1}+1=0 \\
& \Rightarrow 0=C_{1} e-\frac{C_{1}}{e}+1 \\
& \Rightarrow-1=C_{1}\left(e-e^{-1}\right) \\
& \Rightarrow C_{1}=\frac{-1}{\left(e-e^{-1}\right)} \\
& C_{2}=-C_{1}=-\left(\frac{-1}{\left(e-e^{-1}\right)}\right)=\frac{1}{\left(e-e^{-1}\right)}
\end{aligned}
$$

$$
C_{2}=\frac{1}{\left(e-e^{-1}\right)}
$$

STEP 10: The exact solution:

Substitute the values of $C_{1}$ and $C_{2}$ in equation (iv), we get,

$$
\begin{aligned}
& y=\frac{-1}{\left(e-e^{-1}\right)} e^{x}+\frac{1}{\left(e-e^{-1}\right)} e^{-x}+x \\
& y=x-\left[\frac{\left(e^{x}-e^{-x}\right)}{\left(e-e^{-1}\right)}\right]
\end{aligned}
$$

This is the required exact solution.

\section{Conclusion}

This procedure of solving weighted residual method is found to be much simpler than the other methods. Also, it gives an approximate solution and can be compared with exact solution.

\section{Bibliography}

1. Boyd JP. Chebyshev and Fourier spectral methods. Dover;1999.

2. Franklin J. Advanced mechanics and general relativity. Cambridge University Press; 2010.

3. Zienkiewicz OC, Taylor RL. Finite element method. 2000; 1 , The Basis.

4. Cicelia J. Mathematicals method, thermal engineering. S\&S Publication; 2013. 